\title{
Low Frequency Radio Polarization Sensor with Applications in Attitude Estimation
}

\author{
Sean Maguire and Paul Robertson Department of Engineering \\ University of Cambridge \\ Cambridge, UK \\ Email: sean.maguire@cantab.net, par10@cam.ac.uk
}

\begin{abstract}
A novel method of attitude (orientation) estimation is proposed, which makes use of measurements of the polarization of the magnetic (H-) field of Low Frequency (LF) radio signals. This offers advantages relative to existing accelerometer-based systems in high-acceleration, cost-constrained environments such as small fixed-wing Unmanned Aerial Vehicles. In order to validate this method, a sensor system is presented which is capable of determining the $\mathrm{H}$-field polarization of $\mathrm{LF}$ radio signals from three-axis measurements. The sensor system consists of an array of three orthogonal loop antennas, a three channel radio receiver circuit, a datalogging and control module and data processing algorithms. This system is shown to accurately measure the axis of $\mathrm{H}$-field polarization for signals with linear or almost linear polarization, subject to a sign ambiguity. A representative $A M$ radio broadcast in the $L F$ band is shown to have a substantially linear polarization, measurements of which are used to determine a receiver's heading angle. The stability of these polarization measurements over time, and the distorting effect of certain structures, are quantified. The feasibility of this method of attitude determination is therefore demonstrated.
\end{abstract}

Index Terms-Antenna arrays, attitude, low frequency, magnetic sensors, polarization, UAV.

\section{INTRODUCTION}

$\mathbf{M}$ EASUREMENT of a system's attitude (its orientation relative to a reference coordinate frame) is essential in a range of applications, including inertial navigation and attitude control. Within the field of attitude control, there is an increasing requirement for the use of low-cost sensors in challenging environments. For example, small low-cost Unmanned Aerial Vehicles (UAVs) are beginning to be used in applications such as surveying [1], disaster relief [2], search and rescue [3] and agriculture [4]. They require an Attitude and Heading Reference System (AHRS) in order to estimate and control their attitude, but this must also be both small and low-cost.

In a typical low-cost AHRS, MEMS gyroscopes are used to provide an accurate high-bandwidth estimate of angular velocity, which can be integrated (using the appropriate threedimensional kinematic transformation equation [5]) to give angular position and therefore platform attitude relative to the initial orientation. However, changes in the gyroscope bias values over time lead to unbounded errors in the resulting attitude. In order to avoid this drift, the gyroscope measurements are combined with low-bandwidth vector measurements from accelerometers and (often) magnetometers using a filter such as a complementary filter or Extended Kalman Filter
[6]. If it is assumed that a three-axis accelerometer measures the acceleration due to gravity and a three-axis magnetometer measures the Earth's magnetic field, then two vectors can be measured with respect to the sensor axes that are also known with respect to an Earth-fixed coordinate frame. Attitude can thus be resolved as the solution of Wahba's Problem [7], [8], [9], [10], and does not suffer from drift as the vector measurements do not drift. The resulting attitude estimate thus benefits from the gyroscopes' good high-bandwidth performance, while avoiding long term drift.

Such systems have been widely used, but suffer inaccuracies in high-acceleration environments because the accelerometer measures the total (proper) acceleration, which in general includes a dynamic component due to motion in addition to the static and known acceleration due to gravity. For example in fixed-wing UAVs, performing turns may cause extended centripetal accelerations to be experienced. If the accelerometer's measurements are nevertheless assumed to correspond solely to the acceleration due to gravity then large and sustained errors can be introduced into the attitude estimate. Corrections can be applied using data from additional sensors. For example GPS velocity measurements can be used to estimate and correct for the centripetal acceleration [11]. However this provides an indirect measurement, often requires additional assumptions to be made in the aircraft or flight model, and in general relies on an estimate of the local air velocity. It also relies on an accurate GPS signal being available at all times.

An alternative solution is to use different vector inputs to the attitude determination algorithm, either in addition to or instead of the acceleration vector, that are not sensitive to platform acceleration. Suitable vectors must be easily measured with small, light, low-cost and low-power equipment, while also having a known and stable orientation. A candidate for such a vector is a unit vector defined by the polarization of Low Frequency (LF) radio signals. For example, earlier work has demonstrated that the position and orientation of a three-axis LF receiver can be determined relative to a threeaxis transmitter, by sequentially exciting the transmitter with three orthogonal linearly-polarized signals and measuring the response (in the near field) [12], [13].

In this paper a novel sensor is presented which is capable of measuring the polarization of the magnetic field of LF radio signals broadcast from static ground-based transmitters, and which is further suitable in principal for use in small UAVs due to its low size, weight and power consumption. Note 
that while polarization is generally discussed with reference to the electric field, here it is the polarization of the magnetic field that is measured. Section II outlines the properties of LF radio signals and their suitability for this application; section III details the development of a sensor system to measure LF polarization; section IV presents a simple analysis of measurement errors for the LF sensor; and section V presents the results obtained, including analysis of a typical LF signal's polarization, demonstration of heading angle estimation using such signals, and a study of changes in the signals over time and in the presence of distorting structures.

\section{LOW FREQUENCY RADIO}

LF radio signals are defined as having a frequency of 30 - $300 \mathrm{kHz}$. They are used for numerous AM radio broadcasts, navigation beacons, and timing signals. Signals propagate mainly by direct line-of-sight transmission and by groundwave (surface wave) [14]. High power LF transmissions can generally travel very large distances by groundwave. Transmitters are typically vertical monopole antennas with a height somewhat less than a quarter of a wavelength. The vertical transmitters produce waves with substantially linear vertical polarization of the electric field (E-field), and corresponding horizontal polarization of the magnetic field (H-field) perpendicular to both the E-field and the direction of travel. The polarization of the magnetic field is preserved by the transmission mechanisms involved (both direct and groundwave) [15]. This is in contrast to other mechanisms, such as skywave, which can change a signal's polarization properties [14]. It would thus be expected that LF signals can be received over a wide area with a substantially constant and known polarization.

In the general case of an elliptically polarized signal with a receiver in an arbitrary orientation, measurements of an LF signal's H-field in three linearly independent directions allow this polarization to be measured. In the case of substantially linear polarization, as depicted in Fig. 1, this leads to a well defined vector measurement of the major axis of the polarization ellipse, subject to a sign ambiguity. The sign ambiguity can be resolved by also taking E-field measurements, by using an a priori estimate of receiver attitude, or by also measuring a non-orthogonal vector with a known sign, such as the Earth's magnetic field. One or more LF polarization vectors could be used to supplement or replace the accelerometer vector in a traditional AHRS, thus reducing or eliminating its sensitivity to platform acceleration without relying on GPS corrections. The process of using three-axis LF measurements to determine attitude is an extension of traditional Radio Direction Finding.

With reference to Fig. 1, it is useful to define the axial ratio as the length of the major axis of the ellipse divided by the length of the minor axis [14]. The orientation of the major axis can be defined by the angles $\theta$ and $\phi$ in a spherical coordinate system (taking $\theta$ to be measured relative to the horizontal plane for convenience). Here $\phi$ is the "heading" angle of the sensor relative to the major axis and $\theta$ is the "inclination" of the major axis relative to the $x-y$ plane of the sensor. Here all measurements are made with a horizontal sensor for clarity.

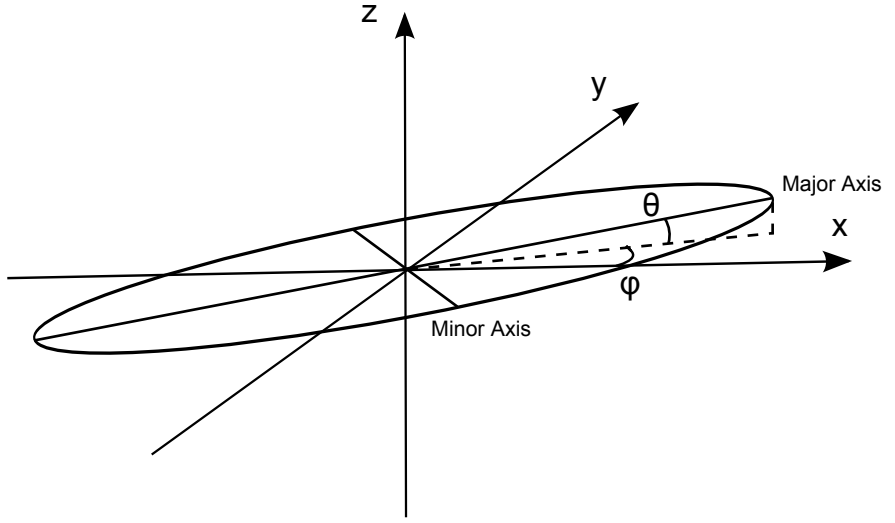

Fig. 1. Diagram of Polarisation Ellipse in 3D Coordinate System, including Definitions of Angles $\theta$ and $\phi$

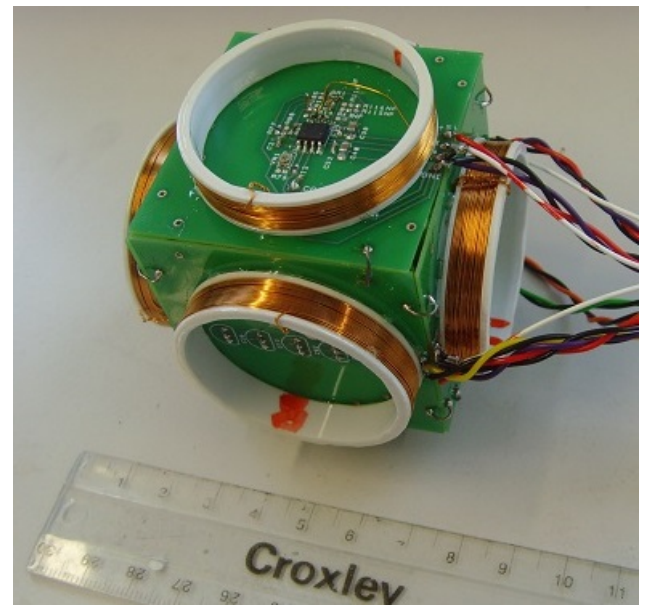

Fig. 3. Receiver Array Comprising Three Orthogonal Loop Antennas

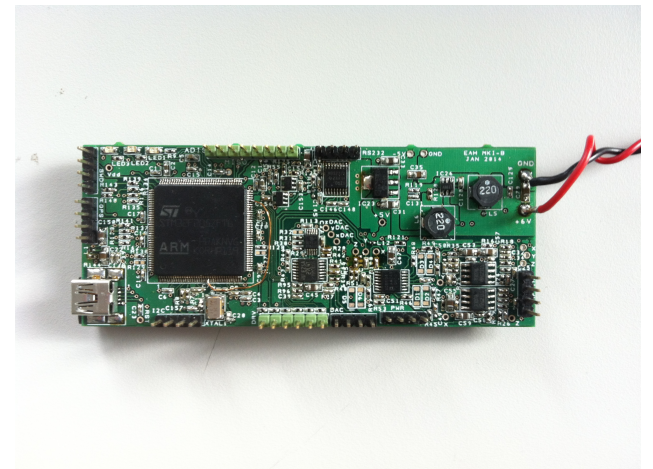

Fig. 4. Datalogging and Control Module

\section{SEnsor DeVElopment}

\section{A. Sensor Hardware}

A small, light and low-cost sensor system has been developed that is capable of measuring the polarization of LF signals. It comprises a novel three-axis LF receiver, a portable datalogging and control module and signal processing algorithms which were implemented off-line. The architecture of the sensor and datalogging hardware is shown in Fig. 2.

The three-axis receiver consists of an array of three or- 


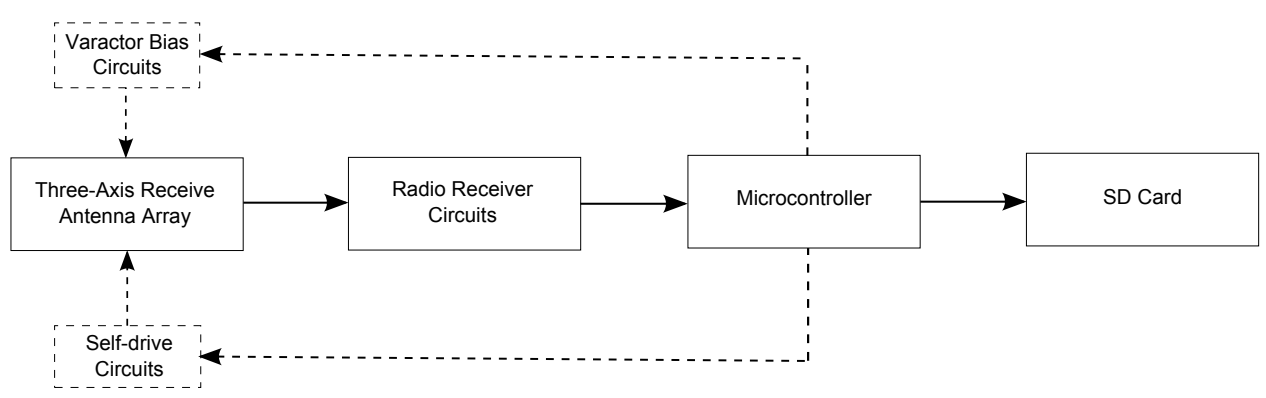

Fig. 2. Block Diagram of Receiver, Including Loop Antenna Array and Processing and Control Element, Showing Main Signal Path (Solid Lines) and Control/Characterization Circuitry (Dashed Lines)

thogonal loop antennas, pictured in Fig. 3, used to sense the instantaneous $\mathrm{H}$-field of an LF signal in three dimensions. Each antenna is constructed from a pair of $40 \mathrm{~mm}$ diameter coils, each consisting of 178 turns of $0.25 \mathrm{~mm}$ diameter copper wire. The coil pairs are connected in series and mounted to opposite faces of a cube to allow a symmetrical array, minimizing cross-coupling between the three axes, to be easily assembled. This arrangement is equivalent to a vector magnetometer. Air-cored coils are used to reduce sensor weight. Electrostatic shielding is used to further reduce cross-coupling. Each antenna coil forms a parallel resonant circuit with a set of $5 \mathrm{KV} 1590 \mathrm{NT}$ varactors, whose capacitance can be varied by the control module. Local "self-drive" signals generated by the control module enable the gain and phase shift of each receiver axis to be individually measured and varied at the frequency of interest. This feature was used to ensure an equal phase shift on each channel, while scale factor corrections were applied in software to account for small differences in gain. The signal from each antenna is directly coupled to a radio receiver circuit including amplifiers and a bandpass filter, before being digitized simultaneously by three Analogue to Digital Converters (ADCs).

The antennas had measured Q-factors at resonance of between 3.8 and 5.2 when tuned to $198 \mathrm{kHz}$, corresponding to an impedance at resonance of between $5.7 \mathrm{k} \Omega$ and 7.8 $\mathrm{k} \Omega$. These low Q-factors are the result using air-cored coils, electrostatic shielding, and weakly-coupled coil pairs. Significant reductions due to the skin and proximity effects were ruled out. While the low Q-factors permit the antennas to be tuned to equalize their phase responses without significantly reducing their amplitude response, a more sensitive system could potentially be obtained by using higher-Q antennas with a different method of phase calibration.

A datalogging and control module, pictured in Fig. 4, was developed in order to record the LF signals detected by the three-axis receiver, in addition to controlling and characterizing the receiver's frequency response. An STM32F205 32bit microcontroller was used to sample the LF signal from the receiver using three on-board simultaneous-sampling 12bit ADCs. A 1 MSPS sampling rate was used to make direct observations of the signal, but the use of a hardware bandpass filter also enabled undersampling at rates as low as $1 \mathrm{kSPS}$, for example used to obtain continuous data over long time periods. A high-speed SD card was used for portable data storage.
Table I summarizes the properties of the prototype sensor. In each case there is scope for further improvement of performance.

TABLE I

PROTOTYPE SENSOR PROPERTIES

\begin{tabular}{cc}
\hline Property & Value \\
\hline Receiver Array Size & $60 \mathrm{~mm} \times 60 \mathrm{~mm} \times 60 \mathrm{~mm}$ \\
\hline Receiver Array Mass & $80 \mathrm{~g}$ \\
\hline Total System Mass & $110 \mathrm{~g}$ \\
\hline Power Consumption & $500 \mathrm{~mW}$ \\
\hline RF Tuning Bandwidth & $60-250 \mathrm{kHz}$ \\
\hline Output Data Rate & $10 \mathrm{~Hz}$ \\
\hline Angle Accuracy & $\pm 4^{\circ}$ \\
\hline
\end{tabular}

\section{B. Measurement Model}

Equations (1) and (2) present a simple measurement model for the sensor. Here $\mathbf{V}_{m}(t)=\left[V_{x}(t) V_{y}(t) V_{z}(t)\right]^{T}$ is the time-varying voltage vector consisting of the amplified and band-limited voltage output from each of the three antennas, $s$ is a scale factor converting from magnetic field strength to voltage, $K$ is a gain matrix containing a scale factor for each axis $\left(k_{11}, k_{22}\right.$ and $\left.k_{33}\right)$ and cross-coupling terms, and $\mathbf{n}(t)=\left[n_{x}(t) n_{y}(t) n_{z}(t)\right]^{T}$ is a noise term. In general the matrix $K$ has a frequency dependence.

$\mathbf{n}(t)$ can be assumed to be additive Gaussian noise with a flat frequency response over a limited bandwidth. The scale factor $s$ does not need to be measured in order to determine signal polarization. The gain matrix $K$ can be measured during calibration. Correcting the measurements for scale factor errors $\left(k_{11}, k_{22}\right.$ and $\left.k_{33}\right)$ was found to marginally improve accuracy. The cross-coupling terms were measured and found to be small. They represent a source of error in the polarization measurements, which is quantified in section IV.

$$
\mathbf{V}_{m}(t)=s K \mathbf{H}(t)+\mathbf{n}(t)
$$




$$
\left[\begin{array}{l}
V_{x}(t) \\
V_{y}(t) \\
V_{z}(t)
\end{array}\right]=s\left[\begin{array}{lll}
k_{11} & k_{12} & k_{13} \\
k_{21} & k_{22} & k_{23} \\
k_{31} & k_{32} & k_{33}
\end{array}\right]\left[\begin{array}{l}
H_{x}(t) \\
H_{y}(t) \\
H_{z}(t)
\end{array}\right]+\left[\begin{array}{l}
n_{x}(t) \\
n_{y}(t) \\
n_{z}(t)
\end{array}\right]
$$

\section{Signal Processing}

The analogue voltage outputs from each receiver circuit $\left(V_{x}(t), V_{y}(t)\right.$ and $\left.V_{z}(t)\right)$ are sampled simultaneously by the ADCs at regular time steps $t=k \tau(k=1,2 \ldots, n)$, resulting in a series of discrete samples given by the vectors $\mathbf{V}_{x}$, $\mathbf{V}_{y}$ and $\mathbf{V}_{z} . \mathbf{V}_{x}$ is shown in (3). $\mathbf{V}_{y}$ and $\mathbf{V}_{z}$ are similarly constructed. These samples are stored by the datalogging and control module and subsequently processed off-line in MATLAB to perform filtering, polarization extraction and attitude determination.

$$
\mathbf{V}_{x}=\left[V_{x}(0) V_{x}(\tau) V_{x}(2 \tau) \ldots V_{x}(n \tau)\right]^{T}
$$

An elliptic Band Pass Filter (BPF) with a bandwidth of $100 \mathrm{~Hz}$ was used to isolate the carrier frequency whose polarization was to be estimated. The same filter was applied to the signal from each axis, resulting in equal changes in the magnitude and phase of the carrier in each case. The filtered measurements $\mathbf{V}_{x}^{\prime}, \mathbf{V}_{y}^{\prime}$ and $\mathbf{V}_{z}^{\prime}$ were then processed to determine the polarization of the LF signal being measured using Singular Value Decomposition.

Equation (4) gives the Singular Value Decomposition of the $n \times 3$ matrix $M$, which contains the filtered measurements as shown in (5).

$$
\begin{gathered}
M=U \Sigma V^{*} \\
M=\left[\begin{array}{ll}
\mathbf{V}_{x}^{\prime} \mathbf{V}_{y}^{\prime} \mathbf{V}_{z}^{\prime}
\end{array}\right]
\end{gathered}
$$

Assuming the rows of $M$ are points on the surface of an ellipsoid, the right singular vectors (the columns of $V$ ) are unit vectors aligned with the axes of the ellipsoid (subject to an unavoidable sign ambiguity), and the singular values (the elements of the diagonal matrix $\Sigma$ ) are the lengths of the axes. This follows from the geometrical interpretation of SVD [16]. The polarization is thus fully determined, and the direction of the major axis is given by the column of $V$ corresponding to the largest singular value. In the presence of noise, the column vectors of $V$ are least squares estimates of the ellipsoid axes (analogous to Principal Component Analysis). This method is routinely used to determine polarization in the field of seismology [17].

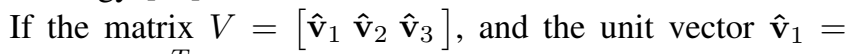
$\left[\begin{array}{lll}\hat{v}_{1 x} & \hat{v}_{1 y} & \hat{v}_{1 z}\end{array}\right]^{T}$ corresponds to the estimated orientation of the major axis of the polarization ellipse, then estimates of the polarization angles $\phi$ and $\theta$ relative to the sensor axes can be obtained from (6) and (7). Similarly, the vector $\hat{\mathbf{v}}_{1}$ is available for use in attitude estimation. Measurements of multiple different radio signals would produce multiple independent vectors.

$$
\begin{gathered}
\hat{\phi}=\arctan \frac{v_{1 y}}{v_{1 x}} \\
\hat{\theta}=\arctan \frac{v_{1 z}}{\sqrt{v_{1 x}^{2}+v_{1 y}^{2}}}
\end{gathered}
$$

\section{ERROR ANALYSIS}

Errors in the raw three-axis measurements of (1) and (2) can lead to errors in the estimated major axis of polarization $\hat{\mathbf{v}}_{1}$ and the corresponding angle estimates $\hat{\theta}$ and $\hat{\phi}$.

Assuming a linearly polarized signal of amplitude $A$ is measured by the sensor, and that the axis of polarization lies in the horizontal plane $(\theta=0)$, the signal's $x-, y$ - and $z$-axis magnetic field is given by (8) - (10) and the major axis of polarization $\mathbf{v}_{1}$ is given by (11). In practice these are reasonable assumptions for the signals in question, where the axial ratio is large and $\theta$ is small.

$$
\begin{aligned}
H_{x}(t) & =A \cos (\phi) \sin (\omega t) \\
H_{y}(t) & =A \sin (\phi) \sin (\omega t) \\
H_{z}(t) & =0 \\
\mathbf{v}_{1} & =\left[\begin{array}{c}
\cos (\phi) \\
\sin (\phi) \\
0
\end{array}\right]
\end{aligned}
$$

Assuming that the noise terms of (1) are zero, and that the axis gains and phase shifts are calibrated such that $k_{11}=$ $k_{22}=k_{33}=1$, errors are due to the cross coupling terms $k_{i j}=\left|k_{i j}\right| e^{\psi_{i j}}(i \neq j)$.

Out of phase coupling leads to errors in the estimate of the minor axis. This error changes the shape of the polarization ellipse, including the axial ratio, but not the orientation of the major axis. The worst case error therefore occurs with in-phase coupling $\left(\psi_{i j}=0\right)$. In this case, under the stated assumptions, the measured analogue voltages are given by (12) and the estimated major axis of polarization is given by (13).

$$
\begin{gathered}
{\left[\begin{array}{c}
V_{x}(t) \\
V_{y}(t) \\
V_{z}(t)
\end{array}\right]=s\left[\begin{array}{ccc}
1 & \left|k_{12}\right| & \left|k_{13}\right| \\
\left|k_{21}\right| & 1 & \left|k_{23}\right| \\
\left|k_{31}\right| & \left|k_{32}\right| & 1
\end{array}\right]\left[\begin{array}{c}
A \cos (\phi) \sin (\omega t) \\
A \sin (\phi) \sin (\omega t) \\
0
\end{array}\right]} \\
\hat{\mathbf{v}}_{1}=\left[\begin{array}{c}
\cos (\phi)+\left|k_{12}\right| \sin (\phi) \\
\left|k_{21}\right| \cos (\phi)+\sin (\phi) \\
\left|k_{31}\right| \cos (\phi)+\left|k_{32}\right| \sin (\phi)
\end{array}\right]
\end{gathered}
$$

This leads to the estimated polarization angles given in (14) and (15), where $C_{\phi}=\cos (\phi)$ and $S_{\phi}=\sin (\phi)$. Assuming $\left|k_{12}\right|<<1$ and $\left|k_{21}\right|<<1$, it follows from (15) that $\hat{\theta} \approx \arctan \left(\left|k_{31}\right| C_{\phi}+\left|k_{32}\right| S_{\phi}\right)$. An upper bound on the error in $\hat{\theta}$ under these assumptions is therefore $\sqrt{2} \arctan \left\{\max \left(\left|k_{31}\right|,\left|k_{32}\right|\right)\right\}$.

Similarly, the worst case error in $\hat{\phi}$ is given by $\arctan \left\{\max \left(\left|k_{21}\right|,\left|k_{12}\right|\right)\right\}$. 


$$
\begin{gathered}
\hat{\phi}=\arctan \left(\frac{\left|k_{21}\right| C_{\phi}+S_{\phi}}{C_{\phi}+\left|k_{12}\right| S_{\phi}}\right) \\
\hat{\theta}=\arctan \left\{\frac{\left|k_{31}\right| C_{\phi}+\left|k_{32}\right| S_{\phi}}{\sqrt{\left(C_{\phi}+\left|k_{12}\right| S_{\phi}\right)^{2}+\left(\left|k_{21}\right| C_{\phi}+S_{\phi}\right)^{2}}}\right\}
\end{gathered}
$$

\section{EXPERIMENTAL RESUlTS}

\section{A. Sensor Characterization}

Cross-coupling between the three receiver axes was measured by applying a signal at the frequency of interest directly to each antenna in turn, with all external signals screened, and measuring the resulting voltages across the other (orthogonal) antennas. At a frequency of $198 \mathrm{kHz}$, the cross-coupling terms of (2) $\left(k_{12}, k_{13}, k_{21}, k_{23}, k_{31}, k_{32}\right)$ were found to have magnitudes of between 0 and 0.05 , with phase angles taking a wide range of values between 0 and $2 \pi$.

The observed coupling factor amplitudes of up to 0.05 would therefore lead to worst-case errors in the measured polarization angles of approximately $\pm 2.9^{\circ}$ in $\hat{\phi}$ and $\pm 4.0^{\circ}$ in $\hat{\theta}$ at $198 \mathrm{kHz}$.

Some measurements were also made at frequencies of 183 $\mathrm{kHz}$ and $162 \mathrm{kHz}$. At these frequencies additional errors are introduced by uncalibrated changes in the magnitude and phase of the axis gains and coupling factors.

The sensor was tested using locally-generated signals in order to verify its operation. A ferrite-cored solenoid coil driven from a signal generator was used to transmit a 198 $\mathrm{kHz} \mathrm{H}$-field signal with approximately linear polarization. The transmitting antenna and the receiver under test were placed 50 $\mathrm{cm}$ apart in an RF screened room in order to eliminate external signals, and the signal measured by the receiver was recorded with the transmitting antenna in different orientations. Agreement between the actual transmitter angle and the measured polarization heading angle was within $7^{\circ}$. This error is likely to be due to both cross-coupling of the signal between the three sensor axes and signal interaction with the metal walls of the screened room.

\section{B. LF Radio Polarisation Measurement}

The sensor was subsequently used to measure an LF signal used for AM broadcasts. BBC Radio 4 longwave, transmitted from Droitwich, UK at $198 \mathrm{kHz}$ with a power of $500 \mathrm{~kW}$, was selected due to its high received signal strength and suitable transmitter characteristics. Measurements were made in Cambridge, UK at a range of approximately $150 \mathrm{~km}$ from the transmitter. As expected, the signal was found to be highly polarized. Fig. 5 shows an example of an observed polarization ellipse, formed by plotting instantaneous twoaxis measurements of the LF signal's H-field sampled at 1 MSPS for a duration of $200 \mu \mathrm{s}$. The measured axial ratio was generally between 8 and 10, indicating almost linear polarization. The ellipse was further found to be oriented in the expected direction, with the major axis close to the horizontal plane $\left(\theta<5^{\circ}\right)$ and close to the perpendicular to the line between transmitter and receiver $\left(\phi<3^{\circ}\right)$, where

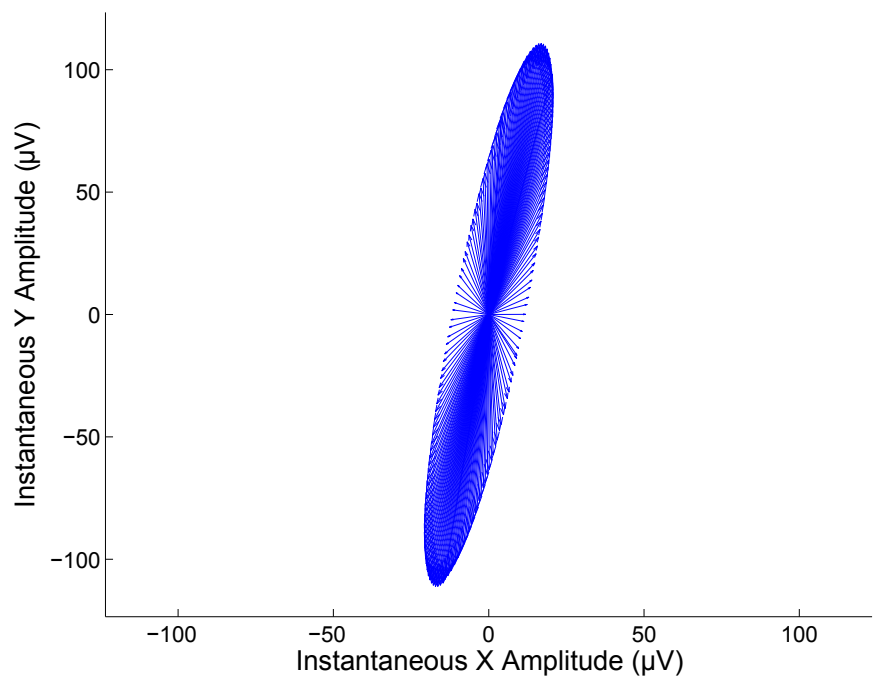

Fig. 5. Typical 2D Polarization Ellipse of Radio 4 Longwave Signal in the Horizontal Plane, Formed as a Vector Plot of the Instantaneous Voltages on two Receiver Axes, Sampled at 1 MSPS over $200 \mu \mathrm{s}$

angles are defined with reference to Fig. $1, z$ is the vertical and $y$ is the direction of travel of the LF wave. These errors can be accounted for by cross-coupling errors of up to $4.0^{\circ}$ and $2.9^{\circ}$ respectively, and slight distortion of the LF signal relative to its expected polarization. Much larger distortions of the polarization were observed when taking measurements near to metal structures or within a few centimeters of the ground, as detailed in section V-D. The observed heading error is consistent with the quoted accuracy of commercial RDF equipment.

In order to verify that this signal can be used to estimate attitude, the receiver was placed in a horizontal position and rotated in the horizontal plane by known angles while measuring the polarization of the $198 \mathrm{kHz}$ Radio 4 carrier signal. As shown in Fig. 6 it was found that the 2D orientation (heading angle) of the receiver could be accurately inferred, subject to a sign ambiguity. The maximum error was $1.6^{\circ}$, which is within the range of errors expected due to crosscoupling between the receiver axes.

Fig. 7 presents heading angle estimates made using three different LF signals during the same experiment, and Fig. 8 shows the magnitude of the errors in each case. The AM stations selected were the $183 \mathrm{kHz}$ Europe 1 station broadcast at 2 MW from Felsberg, Germany and the $162 \mathrm{kHz}$ France Inter station broadcast at $2 \mathrm{MW}$ from Allouis, France, in addition to the $198 \mathrm{kHz}$ Radio 4 signal. The errors in the heading estimates derived from these signals are larger, primarily due to poorer sensor calibration. The longer propagation paths and the lower SNR achieved for the weaker 183 and $162 \mathrm{kHz}$ signals may also have contributed to the errors.

\section{Long Term Signal Stability}

In order to quantify its long term stability, the $198 \mathrm{kHz}$ BBC Radio 4 signal was measured over a 24 hour period, sampled at $1.12 \mathrm{kSPS}$, and estimates of the orientation of the major axis of the polarization ellipse were calculated at one 


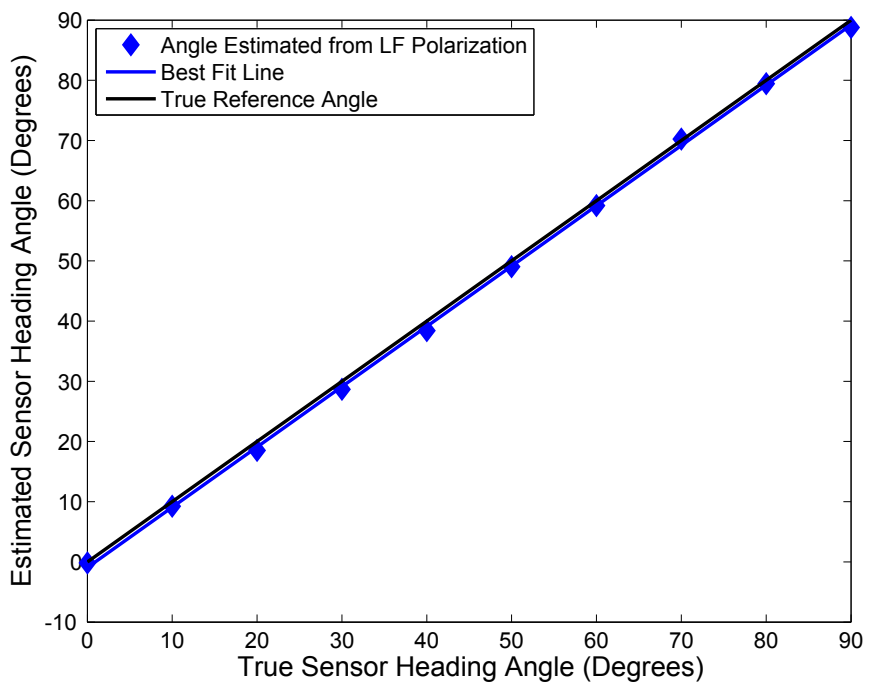

Fig. 6. Estimated Relative Heading Angle $\hat{\phi}$ based on LF Polarization of 198 kHz Radio 4 Signal, Plotted Against Measured Angle for a Ninety Degree Rotation in the Horizontal Plane

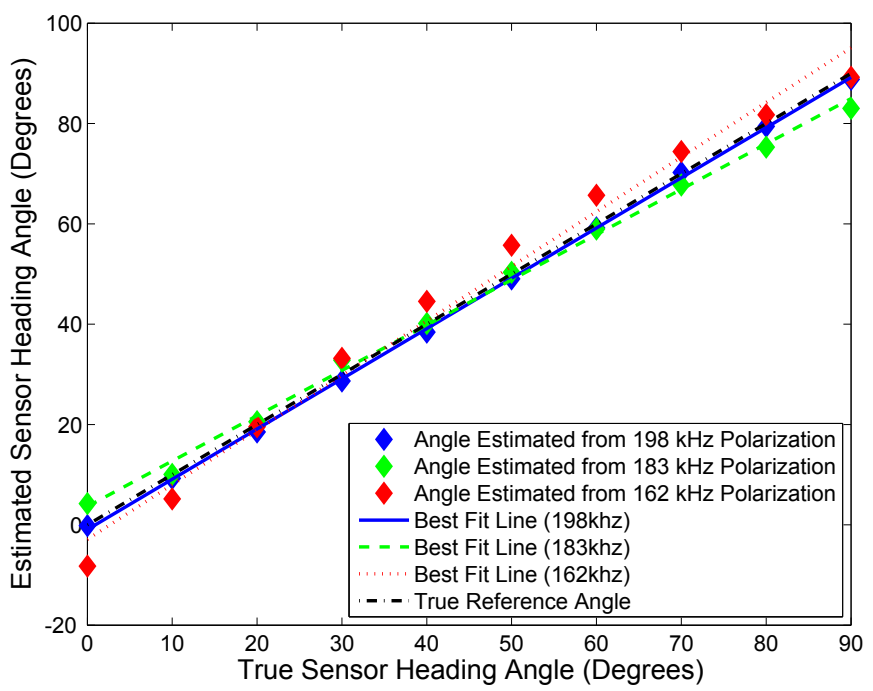

Fig. 7. Relative Heading Angles Estimated Independently from Polarization of Three LF Signals, Plotted Against Measured Angle for a Ninety Degree Rotation in the Horizontal Plane

second intervals (subject to the sign ambiguity). Fig. 9 shows a histogram of measurements of $\theta$ over the 24 hour period, while similar results were obtained for $\phi$, indicating that the polarization estimates are reliable over a period of 24 hours.

The signal properties were found to differ substantially between night and day. Although the measurements in both cases have similar means, the variance is significantly higher at night. This is likely to be the result of changes in the RF propagation environment. In particular, skywave propagation is more effective at night, and does not preserve signal polarization [14]. It would be expected that the dominant received LF signal is due to direct line of sight transmission and groundwave propagation, and therefore has a well-defined polarization, but that a small component of the received signal is due to skywave propagation and has a poorly-defined

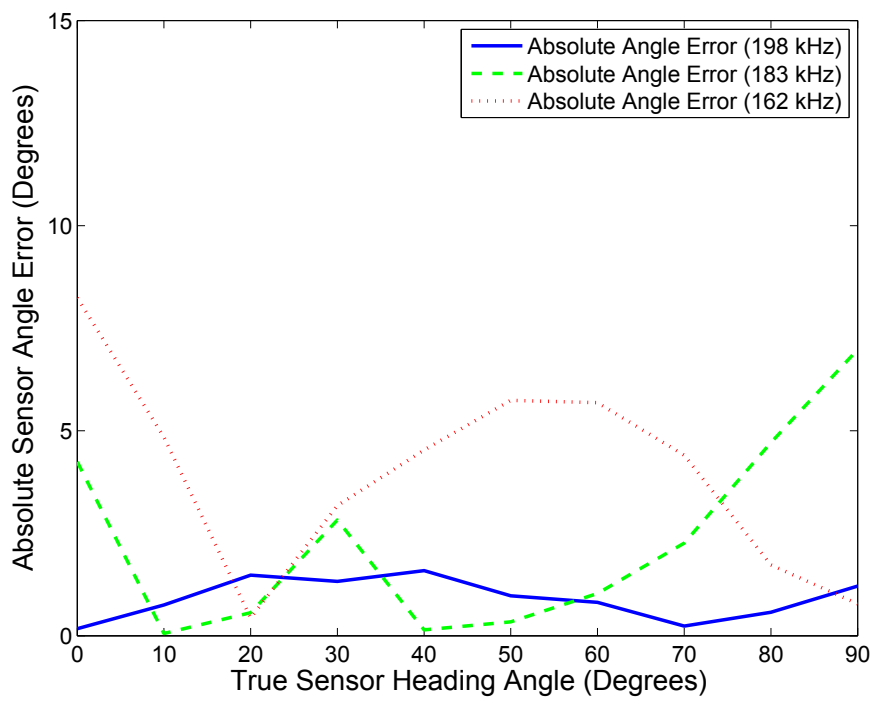

Fig. 8. Error Between Estimated and True Relative Heading Angles for a Ninety Degree Rotation in the Horizontal Plane

polarization. It would further be expected that this component would be larger at night, giving rise to a larger variance of the polarization angles at night as observed. In addition to affecting the propagation of the LF signal of interest, there are other more distant stations broadcasting on the same frequency $(198 \mathrm{kHz})$ which are likely to be more strongly received at long range by night, and thus to cause greater interference. This increase in variance would reduce the useful sensor bandwidth at night.

Further measurements were made over a period of seven days. Fig. 10 shows the heading angle estimate based on the 198 kHz BBC Radio 4 carrier signal, with nights (9pm-9am) highlighted in gray. Note that this data has been low pass filtered using a filter with a cut-off frequency of $0.1 \mathrm{~Hz}$ in order to remove noise, and that outliers have been removed. Tables II and III summarize the mean and standard deviation of the (unfiltered) polarization angles measured during each 12 hour period.

These results demonstrate that there is little variation in the long term mean values of the polarization over time, but that the standard deviation is significantly higher at night. They also demonstrate that the standard deviation of the heading angle $\phi$ is significantly larger than the standard deviation of the inclination angle $\theta$. This is consistent with signal components with a horizontally polarized E-field being attenuated by the conductive ground.

\section{Signal Distortion}

Significant distortion of the LF signals was observed when the sensor was in close proximity to vertically orientated conductive loops, with particularly large errors in inclination angle estimate $\hat{\theta}$.

The distortion caused by a triangular aluminum frame, with side lengths of $1.25 \mathrm{~m}, 1.25 \mathrm{~m}$ and $1.0 \mathrm{~m}$, was analyzed by placing the frame in a vertical orientation at varying distances from the sensor. Fig. 12 shows the resulting inclination angle 


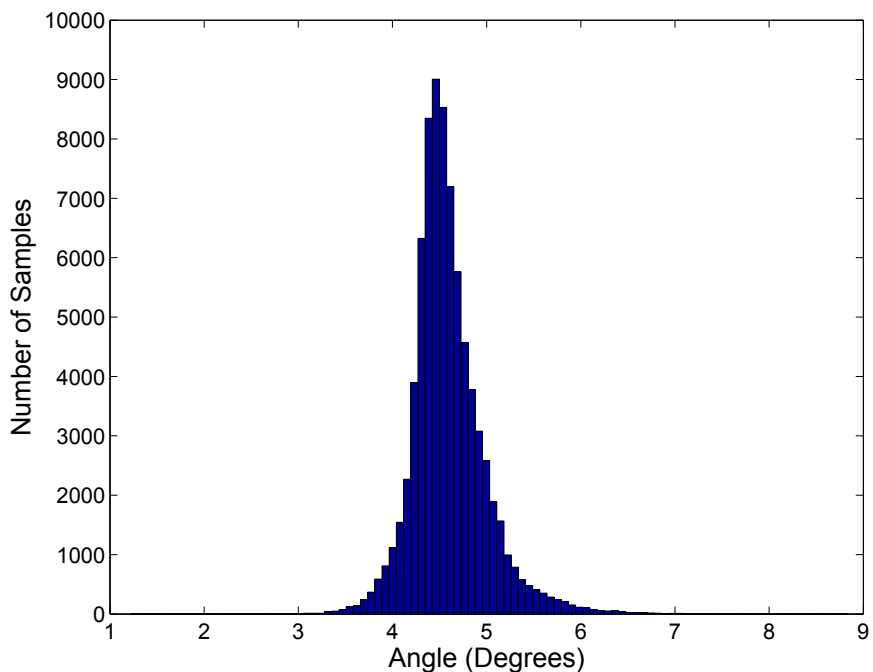

Fig. 9. Histogram of Estimated Inclination Angle $\hat{\theta}$ based on $198 \mathrm{kHz}$ BBC Radio 4 Signal, easured over 24 Hours

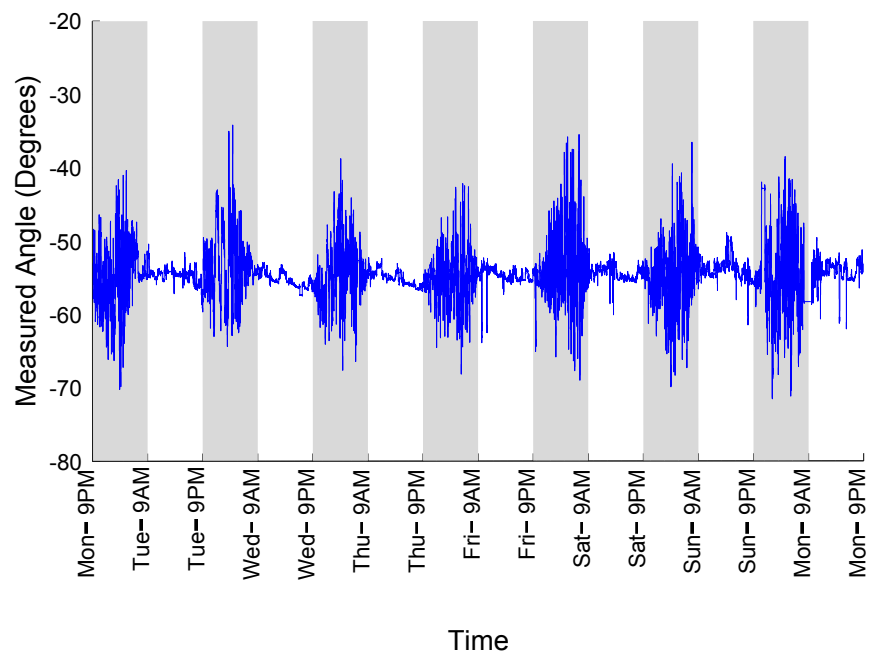

Fig. 10. Estimated Heading Angle $\hat{\phi}$ based on $198 \mathrm{kHz}$ BBC Radio 4 LF Polarization over One Week (Highlighting Night Time Periods)

TABLE II

Mean and Standard Deviation of Polarization Angle Estimates ON SEVEN CONSECUTIVE DAYS

\begin{tabular}{ccccc}
\hline Day & Mean $(\hat{\boldsymbol{\theta}})$ & $\begin{array}{c}\text { Standard } \\
\text { Deviation } \\
(\hat{\boldsymbol{\theta}})\end{array}$ & Mean $(\hat{\boldsymbol{\phi}})$ & $\begin{array}{c}\text { Standard } \\
\text { Deviation } \\
(\hat{\boldsymbol{\phi}})\end{array}$ \\
\hline Tuesday & $4.0^{\circ}$ & $0.3^{\circ}$ & $-54.7^{\circ}$ & $1.2^{\circ}$ \\
\hline Wednesday & $4.0^{\circ}$ & $0.4^{\circ}$ & $-55.2^{\circ}$ & $1.1^{\circ}$ \\
\hline Thursday & $3.7^{\circ}$ & $0.4^{\circ}$ & $-55.1^{\circ}$ & $1.1^{\circ}$ \\
\hline Friday & $3.5^{\circ}$ & $0.3^{\circ}$ & $-54.5^{\circ}$ & $1.0^{\circ}$ \\
\hline Saturday & $3.4^{\circ}$ & $0.4^{\circ}$ & $-54.3^{\circ}$ & $1.2^{\circ}$ \\
\hline Sunday & $3.7^{\circ}$ & $0.4^{\circ}$ & $-53.8^{\circ}$ & $1.8^{\circ}$ \\
\hline Monday & $3.3^{\circ}$ & $0.3^{\circ}$ & $-54.0^{\circ}$ & $1.5^{\circ}$ \\
\hline
\end{tabular}

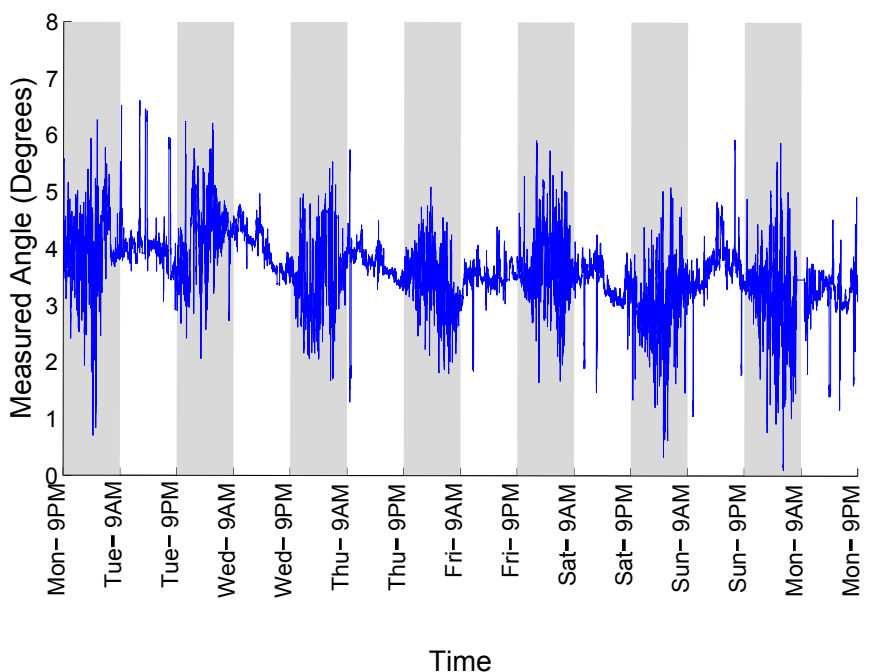

Fig. 11. Estimated Inclination Angle $\hat{\theta}$ based on 198kHz BBC Radio $4 \mathrm{LF}$ Polarization over One Week (Highlighting Night Time Periods)

TABLE III

Mean and Standard Deviation of Polarization Angle Estimates on SEVEN CONSECUTIVE NightS

\begin{tabular}{ccccc}
\hline Night & Mean $(\hat{\boldsymbol{\theta}})$ & $\begin{array}{c}\text { Standard } \\
\text { Deviation } \\
(\hat{\boldsymbol{\theta}})\end{array}$ & Mean $(\hat{\boldsymbol{\phi}})$ & $\begin{array}{c}\text { Standard } \\
\text { Deviation } \\
(\hat{\boldsymbol{\phi}})\end{array}$ \\
\hline Monday & $4.0^{\circ}$ & $0.7^{\circ}$ & $-54.9^{\circ}$ & $4.5^{\circ}$ \\
\hline Tuesday & $4.2^{\circ}$ & $0.7^{\circ}$ & $-53.3^{\circ}$ & $4.4^{\circ}$ \\
\hline Wednesday & $3.5^{\circ}$ & $0.7^{\circ}$ & $-54.9^{\circ}$ & $3.9^{\circ}$ \\
\hline Thursday & $3.4^{\circ}$ & $0.6^{\circ}$ & $-55.2^{\circ}$ & $3.7^{\circ}$ \\
\hline Friday & $3.6^{\circ}$ & $0.6^{\circ}$ & $-53.5^{\circ}$ & $4.4^{\circ}$ \\
\hline Saturday & $3.0^{\circ}$ & $0.7^{\circ}$ & $-55.0^{\circ}$ & $4.3^{\circ}$ \\
\hline Sunday & $3.1^{\circ}$ & $0.8^{\circ}$ & $-55.2^{\circ}$ & $5.1^{\circ}$ \\
\hline
\end{tabular}

$\theta$ of three LF signals when the frame was placed at distances of between 0 and $25 \mathrm{~cm}$ from the sensor. It can be seen that very large errors were introduced when the frame was in close proximity to the sensor, but that these reduced rapidly as the distance between the frame and sensor increased. The rate of decay of the magnitude of the spurious signal is close to a $1 / r$ relationship over this distance scale.

This effect is likely to be due to the conductive frame acting as a loop antenna and re-radiating a non-uniform local $\mathrm{H}$-field. It complicates the use of this technique close to metal-framed vehicles or structures.

\section{CONCLUSION}

A novel three-axis LF radio polarization sensor has been demonstrated and shown to accurately measure the axis of $\mathrm{H}$-field polarization for LF signals with approximately linear polarization. The sensor has been used to measure the polarization of three widely available LF radio signals, and the observed polarization was found to agree with that predicted 


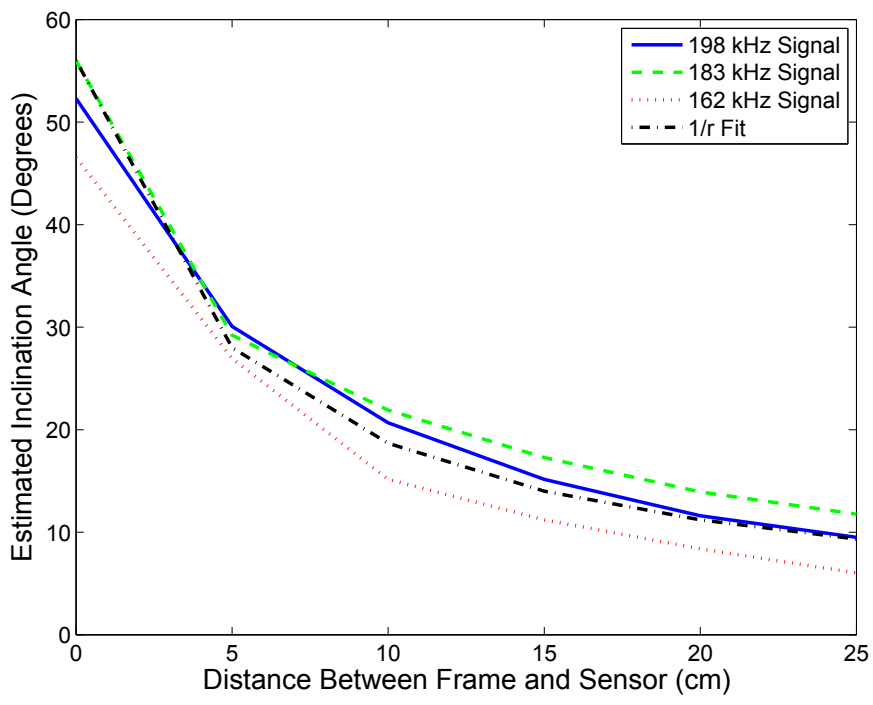

Fig. 12. Estimated Inclination Angles $\hat{\theta}$ based on Three LF Signals in the Presence of a Vertical aluminum Frame at Varying Distances from the Sensor, with Simulated $1 / r$ Curve

by theory. The agreement was particularly close for measurements at the calibration frequency, using the strongest and closest transmitter. Heading determination has been demonstrated using these polarization measurements, subject to a sign ambiguity. The same approach can also be used to determine pitch and roll given observations of at least two non-collinear LF polarization vectors. In addition, the orientation of the polarization axis of a typical signal was found to be stable over time, although with a higher variance at night. These results support the assertion that such a sensor could be used to supplement or replace the accelerometer in an AHRS in high-acceleration cost-constrained environments, in order to reduce its sensitivity to dynamic acceleration.

Further work is needed to demonstrate real-time attitude determination using the sensor, to validate its operation in specific applications such as a small UAV AHRS and to evaluate the performance improvement offered by including this sensor as an AHRS input. Improvements to the sensor's properties (including size, weight, bandwidth, power consumption and accuracy) could also be achieved through further development.

\section{REFERENCES}

[1] S. Siebert and J. Teizer, "Mobile 3D mapping for surveying earthwork projects using an unmanned aerial vehicle (UAV) system," Automation in Construction, vol. 41, pp. 1 - 14, May 2014.

[2] H. Bendea, P. Boccardo, S. Dequal, F. Giulio-Tonolo, D. Marenchino, and M. Piras, "Low cost UAV for post-disaster assessment," in The Int. Archives of the Photogrammetry, Remote Sensing and Spatial Information Sci., vol. 37, no. B8, Beijing, China, July 2008, pp. 1373 1379.

[3] M. A. Goodrich, B. S. Morse, D. Gerhardt, J. L. Cooper, M. Quigley, J. A. Adams, and C. Humphrey, "Supporting wilderness search and rescue using a camera-equipped mini UAV," J. Field Robotics, vol. 25, no. 1-2, pp. 89-110, January-February 2008.

[4] J. Berni, P. J. Zarco-Tejada, L. Suarez, and E. Fereres, "Thermal and narrowband multispectral remote sensing for vegetation monitoring from an unmanned aerial vehicle," IEEE Trans. Geosci. Remote Sens., vol. 47, no. 3, pp. $722-738$, March 2009.
[5] W. F. Phillips, C. E. Hailey, and G. A. Gebert, "Review of attitude representations used for aircraft kinematics," J. Aircraft, vol. 38, no. 4, pp. 718-737, July-August 2001.

[6] D. Gebre-Egziabher, R. C. Hayward, and J. D. Powell, "Design of multisensor attitude determination systems," IEEE Trans. Aerosp. Electron. Syst., vol. 40, no. 2, pp. 627-649, April 2004.

[7] J. L. Farrell, J. C. Stuelpnagel, R. H. Wessner, and J. R. Velman, "A least squares estimate of satellite attitude," SIAM Review, vol. 8, no. 3, pp. 384-386, July 1966.

[8] M. D. Shuster and S. D. Oh, "3-axis attitude determination from vector observations," J. Guidance and Control, vol. 4, no. 1, pp. 70-77, January-February 1981.

[9] F. L. Markley and D. Mortari, "Quaternion attitude estimation using vector observations," J. Astronaut. Sci., vol. 48, no. 2-3, pp. 359-380, April-September 2000.

[10] F. L. Markley, "Attitude determination using vector observations and the singular value decomposition," J. Astronaut. Sci., vol. 36, no. 3, pp. 245-258, July-September 1988.

[11] M. Euston, P. Coote, R. Mahony, J. Kim, and T. Hamel, "A complementary filter for attitude estimation of a fixed-wing UAV," in Proc. 2008 IEEE/RSJ Int. Conference on Intell. Robots and Syst., Nice, France, September 2008, pp. 340-345.

[12] F. H. Raab, E. B. Blood, T. O. Steiner, and H. R. Jones, "Magnetic position and orientation tracking system," IEEE Trans. Aerosp. Electron. Syst., vol. 15, no. 5, pp. 709-718, September 1979.

[13] F. H. Raab, "Quasi-static magnetic-field technique for determining position and orientation," IEEE Trans. Geosci. Remote Sens., vol. 19, no. 4, pp. 235-243, October 1981.

[14] J. S. Seybold, Introduction to RF Propagation. New York: John Wiley \& Sons, 2005

[15] K. A. Norton, "The physical reality of space and surface waves in the radiation field of radio antennas," Proc. Inst. Radio Eng., vol. 25, no. 9, pp. 1192-1202, September 1937.

[16] G. Strang, Linear Algebra and its Applications, 4th ed. WB Saunders, 2006.

[17] R. de Franco and G. Musacchio, "Polarization filter with singular value decomposition," Geophysics, vol. 66, no. 3, pp. 932-938, May-June 2001.

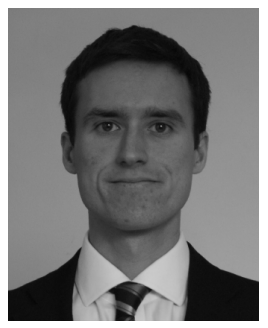

Sean Maguire received the M.Eng. degree in Electrical and Information Sciences from the University of Cambridge, Cambridge, UK in 2011, and subsequently joined the Photonics and Sensors Group at the University of Cambridge to pursue the Ph.D. degree. His research interests include Low Frequency electromagnetic sensors and low-cost attitude determination systems.

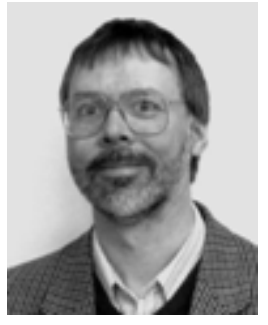

Paul Robertson received the BA degree in Electrical Sciences from the University of Cambridge, Cambridge, UK in 1984 followed by a Ph.D. on thin film electronics in 1987 from the same institution.

From 1987 to 1993 he worked at the PA Consulting Group technology center in Melbourn before returning to the faculty in the Engineering Department at the University of Cambridge. He is author of over 50 technical papers and patents. His current research interests include electromagnetic sensors for instrumentation, hybrid-electric propulsion systems for aircraft and power generating systems. He has been a Chartered Engineer and a Member of the Institution of Engineering and Technology (IET) since 1993. 